\title{
General Introduction to Part Two
}

This part contains three research papers reprinted from academic journals. The first two papers make direct use of path analysis; the third deals with an application of regression analysis.

The papers have been chosen to illustrate the diverse uses to which causal analysis can be put. In the preceding pages, the theory underlying causal analysis has been examined and a simple hypothetical example has been discussed. The purpose of the subsequent three chapters is to show how this theory can be put to real practical use.

None of the papers makes use of the likelihood method of hypothesis testing, illustrated in the example presented in the last section of Part $l$. This is because, to date, real examples of the use of this technique are rare. The reader is urged to examine in which respects the use of the likelihood test would benefit the analysis in each of these papers - in this way he will greatly increase his understanding of the theory and its practical implications.

The fact that these papers do not use the more sophisticated methods of statistical inference discussed in Part 1 should in no way detract from their merits. In each one, careful consideration is given to measurement and to specification problems, and a careful analysis is made of the theoretical implications of the quantitative results. Imaginative use is made of causal analysis, and, in many ways, this far outweighs the absence of the more sophisticated inferential techniques. If these papers go some way to teaching the reader how to use causal analysis creatively then this knowledge, coupled with the theoretical skills gained in Part 1 , should allow him to make the best use of causal analysis in whatever fields of research he wishes to pursue. 